\section{Visión Electrónica \\ Más que un estado sólido}

https://revistas.udistrital.edu.co/index.php/visele

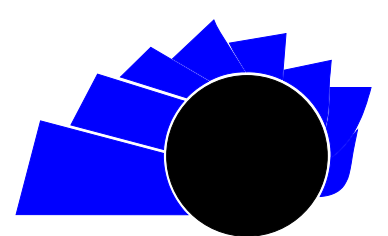

VISIÓN ELECTRÓNICA

VISIÓN INVESTIGADORA

\title{
Diseño de red óptica de larga distancia reconfigurable
}

\author{
Design of Configurable Long Reach Optical Network
}

Sergio Alejandro Pérez Almeida. ${ }^{1}$, Gustavo Adolfo Puerto Leguizamón. ${ }^{2}$

INFORMACIÓN DEL ARTÍCULO

Historia del artículo:

Enviado: 23/10/2017

Recibido: $11 / 11 / 2017$

Aceptado: 14/01/2018

\section{Palabras clave:}

AWG

PON

Presupuesto de ancho de banda

Presupuesto de potencia

Switches ópticos

WDM

\section{Open access}

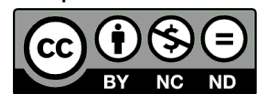

\section{Keywords:}

AWG

PON

Optical bandwidth budget

Optical power budget

Optical switches

WDM

\section{RESUMEN}

Actualmente, las redes de fibra óptica están en proceso de implementación en todo el mundo ya que poseen ventajas frente a las redes tradicionales, entre ellas el aumento de la cobertura y altas velocidades de transmisión; sin embargo, las redes de acceso de fibra óptica para largas distancias son ahora un tema de investigación, debido a que el aumento de las distancias involucra factores limitantes como el de la atenuación y la dispersión, los cuales degradan el rendimiento de la red. En este artículo de investigación se propone el diseño de una red óptica de larga distancia con un enfoque PON (Passive Optical Network) y capacidades multicanal, la cual es reconfigurable por medio de switches ópticos y AWG (Arrayed Waveguide Gratings). Se indican los cálculos realizados, así como el diseño de los nodos de distribución. Finalmente, el sistema es simulado y analizado para determinar la viabilidad de la red.

\section{ABSTRACT}

Nowadays the optical fibers networks are in the process of implementation around the world, this networks have many advantages like better coverage and higher transmission velocity of information than the others; however, the long reach optical networks are still under studies, due to the increase of the distances we must consider limiting factors like attenuation or dispersion of the signal that degrade the network performance. In this researched paper, we proposed the design of a long reach optical network with a PON (Passive Optical Network) approach and multichannel capacities, which is configurable through the optical switches and AWGs (Arrayed Waveguide Gratings). We do the estimates, design of distribution nodes. Finally, the optical system is simulated to determinate the viability of the network.

\footnotetext{
${ }^{1}$ Ingeniero electrónico, Universidad Distrital Francisco José de Caldas. Magíster en Ciencias de la Información y las Comunicaciones, Universidad Distrital Francisco José de Caldas. Correo electrónico: sapereza@correo.udistrital.edu.co

${ }^{2}$ Ingeniero de Telecomunicaciones. Docente Facultad de Ingeniería Universidad Distrital Francisco José de Caldas. Doctor en Telecomunicaciones Universidad Politécnica de Valencia, España. gapuerto@udistrital.edu.co
} 


\section{Introducción}

En la actualidad, el acceso a internet ha crecido de manera vertiginosa. Según estadísticas de la población que tiene acceso a internet en el ámbito mundial, encontradas en la Internet World Stats [1], se nota una tendencia al crecimiento. Para el caso de Latinoamérica, desde el 2000 hasta el 2017 se tuvo un aumento de la población con acceso a internet de alrededor de $2035 \%$.

Además, aparte del reto de garantizar la infraestructura adecuada para el acceso de los usuarios a Internet, también se debe atender la creciente demanda de tasas de transmisión más elevadas que son necesarias para los nuevos servicios ofrecidos, entre los cuales se encuentran: transmisión de video de alta definición, transferencia punto a punto de grandes archivos, vídeos bajo demanda, juegos en línea, entre otros. Según proyecciones de la empresa Cisco Systems, presentados en la Figura 1, la cantidad de tráfico IP por mes creció de 59.9 exabytes en el 2014 a 168 exabytes para el 2019, [2]; y, por supuesto, la infraestructura de red deberá estar disponible para soportar tal cantidad de tráfico.

Figura 1: Crecimiento en el tráfico de internet proyectado para el 2019 [2].

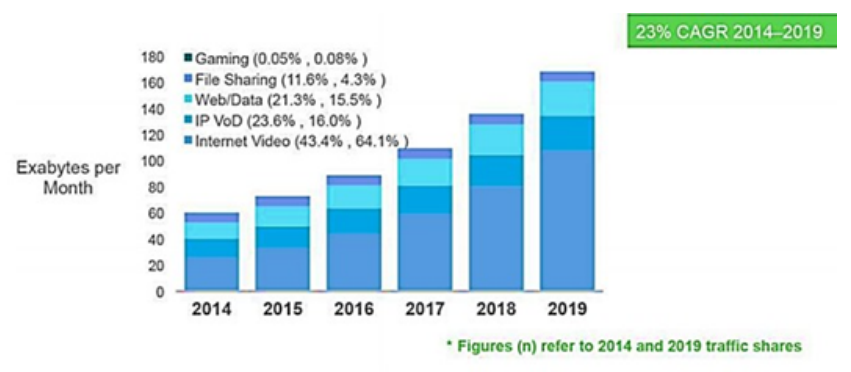

Source: Cisco VNI Global P Trattc Forecast, 2014-2019

Por otra parte, hoy en día las redes de comunicación se componen por la red troncal que básicamente es el Backbone - ruta principal para salir a internet-, la cual ya provee gran capacidad de ancho de banda; posteriormente, se encuentra la red metro que, según su topología, conecta toda la infraestructura de red de una ciudad o institución; finalmente, se encuentra la red de acceso, la cual es la red que provee conectividad directa al usuario hacia otras redes. Típicamente, las tecnologías utilizadas en la red de acceso son: el acceso a internet por cable coaxial y DSL (Digital Subcriber Line) [3] junto a sus derivaciones; sin embargo, estas tecnologías se han convertido en un cuello de botella, pues tienen grandes limitaciones respecto a la capacidad de tráfico ofrecido y su longitud máxima de funcionamiento, por lo que los operadores de internet están migrando a soluciones compuestas por redes de acceso en fibra óptica. En ese sentido, mundialmente se han empezado a implementar las redes de acceso ópticas pasivas denominadas PON (Passive Optical Network), las cuales eliminan cuello de botella gracias al alcance y ancho de banda que pueden ofrecer; adicionalmente, se están estudiando las redes de largo alcance PON - denominadas Long Reach $\mathrm{PON}$ - para extender la longitud de funcionamiento de 20 kilómetros hasta los 100 kilómetros o más [4].

Las redes LR-PON (Long Reach PON) son redes ópticas de largo alcance, estas redes aún no se encuentran estandarizadas y están en proceso de investigación, ya que el aumento de las distancias genera nuevos desafíos en cuanto a superar la atenuación refiriéndose a la primera capa del modelo OSI, también generan desafíos en cuanto al proceso para el establecimiento de la comunicación y manejo de tramas perdidas entre el OLT (Optical Line Termination) y las ONU (Optical Network Unit).

Figura 2: Red PON convención versus LR-PON [5].

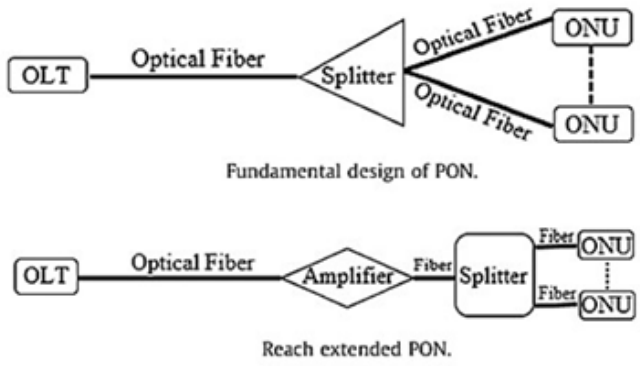

En la Figura 2 se pueden apreciar dos esquemas básicos de las redes PON convencionales y las redes LR-PON. La principal diferencia entre en los dos tipos de red son las distancias de cobertura, los estándares PON convencionales (E-PON, GPON, entre otras) [6] especifican una distancia entre el OLT y las ONU de máximo $20 \mathrm{~km}$, mientras que las redes LR-PON están pensadas para alcanzar distancias de hasta $100 \mathrm{~km} \mathrm{o}$ más; este aumento de cobertura hace que sea necesario amplificar la señal enviada y recibida, dejando de ser una red pasiva. Estos esquemas de amplificación también se encuentran en investigación actualmente [7].

En $[4,8,9]$ y [10] se observan diferentes propuestas de diseño para redes de acceso ópticas de larga distancia, en estos artículos se puede observar el uso de elementos pasivos y activos, así como la inclusión de la multiplexación por longitud de onda WDM (Wavelength Division Multiplexing). 
Para el diseño de una red de acceso óptica de largo alcance se debe tener en cuenta qué tipo de red se quiere realizar, ya que la inclusión de elementos como los AWG y los switches ópticos permiten una alta flexibilidad en el diseño; si se quiere un esquema estático es necesario el uso de AWG o cualquier otro filtro óptico como en el caso de la Figura 3.

Figura 3: Aplicaciones de los filtros para crear un sistema de longitudes de onda entrecruzadas (wavelength crossconnect) [11].

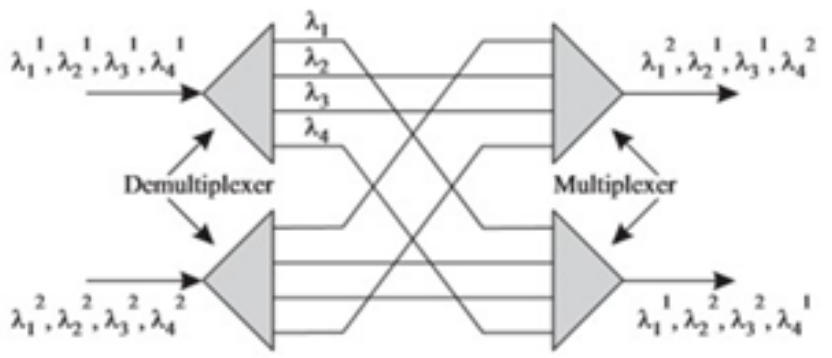

Para esquemas dinámicos se tiene se considera la Figura 4.

Figura 4: Aplicaciones de los filtros para crear un sistema de longitudes de onda entrecruzadas dinámicas [11].

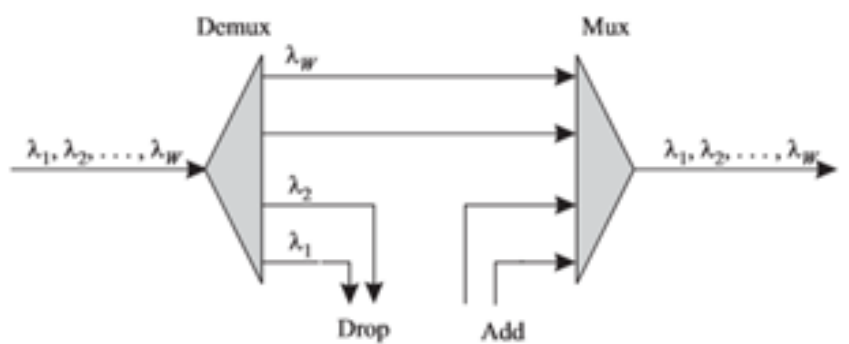

Como se puede analizar en el esquema dinámico, el uso del AWG unido a conmutadores ópticos u otros dispositivos agregan flexibilidad a la red, ya que permite la adición y sustracción de canales con diferentes longitudes de onda y a su vez permite la reconfiguración para el enrutamiento según se requiera.

Hay que recordar que estos dispositivos son elementos que funcionan en el dominio óptico, es decir, en ningún momento hay que realizar conversiones entre el dominio eléctrico y el óptico, lo cual favorece la obtención de mayores anchos de banda; también las redes ópticas de acceso de larga distancia se basan en redes PON, sin embargo, el agregar un factor distancia en el diseño implica que se deban utilizar amplificadores ópticos y elementos compensadores de dispersión, para garantizar el presupuesto de potencia y el ancho de banda respectivamente.

El artículo se estructura de la siguiente manera: primero se definen los materiales y métodos para el diseño de la red, incluyendo el presupuesto de potencia y ancho de banda y el diseño de puntos de distribución; luego se realiza la simulación de la red utilizando Optisystem; posteriormente, se establecen los resultados en términos de potencia de las señales recibidas y diagramas de ojo junto con su discusión; finalmente, se dan las conclusiones.

\section{Diseño de una red de acceso óptica de larga distancia}

En primera instancia, para realizar un diseño se deben tener los datos básicos de la red: distancia de cobertura, plan de frecuencias, ancho de banda, tipo de fibra a utilizar, entre otros; para este diseño en específico se utilizarán los dados en la Tabla 1.

Luego, se contemplaron cuatro poblaciones, las cuales dispondrán cada una de una longitud de onda fija y dos longitudes de onda de respaldo para toda la red, es decir, si en alguna ubicación hay un consumo excesivo de ancho de banda el sistema estará en la capacidad de redirigir las longitudes de ondas auxiliares a dicha ubicación.

En la Figura 5 se pueden visualizar cuatro tramos ópticos (Tabla 2).

Figura 5: Red por diseñar.

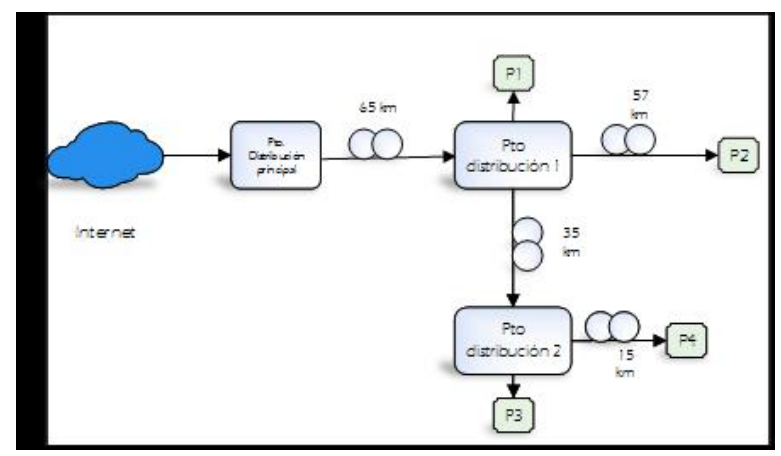

Fuente: elaboración propia. 
Tabla 1: Parámetros de elementos considerados en la red óptica.

\begin{tabular}{|c|c|c|c|}
\hline Transmisor & $\begin{array}{c}\text { Puntos de } \\
\text { distribución }\end{array}$ & Tramo óptico & Receptor \\
\hline Laser CW & AWG & Fibra óptica & Fotodetector APD \\
\hline Potencia $=15 \mathrm{dBm}$ & Mux/Demux & ITU-T G.652.D & Ganancia $=3$ \\
\hline $\begin{array}{l}\text { Frecuencia = } \\
192.8-193.2\end{array}$ & $\begin{array}{l}\text { Ancho de banda= } \\
100 \mathrm{GHz}\end{array}$ & Región= $1550 \mathrm{~nm}$ & Responsividad $=1 \mathrm{~A} / \mathrm{W}$ \\
\hline \multirow[t]{2}{*}{$\begin{array}{l}\text { Ancho de línea = } \\
10 \mathrm{Mhz}\end{array}$} & $\mathrm{IL}=4 \mathrm{~dB}$ & $\begin{array}{l}\text { Dispersión = } 17 \\
\mathrm{ps} / \mathrm{nm} \cdot \mathrm{km}\end{array}$ & Dark current $=10 \mathrm{nA}$ \\
\hline & Filtro= Gausiano & $\mathrm{A}=0.2 \mathrm{~nm} / \mathrm{km}$ & \\
\hline $\begin{array}{l}\text { Pseudo Ramdom } \\
\text { Bit }\end{array}$ & orden $=2$ & Carrete $=5 \mathrm{~km}$ & Filtro pasa bajo de bessel \\
\hline \multirow[t]{2}{*}{$10 \mathrm{Gbps}$} & & & $\begin{array}{l}\text { frecuencia de corte }=0.75 \\
* \text { Bit rate }\end{array}$ \\
\hline & Switch óptico & $\begin{array}{l}\text { Empalmes y } \\
\text { Conectores }\end{array}$ & \\
\hline $\begin{array}{l}\text { Generado de pulsos } \\
\text { NRZ }\end{array}$ & MEMS & $\mathrm{IL}=0.1 \mathrm{~dB}$ & AWG \\
\hline (No retorno a cero) & $1 \mathrm{xN}$ & & Demux \\
\hline $\begin{array}{l}\text { Modulador Mach- } \\
\text { Zehnder }\end{array}$ & $\mathrm{IL}=0.8 \mathrm{~dB}$ & $\begin{array}{l}\text { Fibra DCF } \\
\text { DRAKA [12] }\end{array}$ & $\begin{array}{l}\text { Ancho de banda }=100 \\
\mathrm{GHz}\end{array}$ \\
\hline $\begin{array}{l}\mathrm{IL}=3 \mathrm{~dB} \\
\text { aproximadamente }\end{array}$ & $\begin{array}{l}\text { crosstalk }-50 \mathrm{~dB} \\
\text { máx. }\end{array}$ & & $\mathrm{IL}=4 \mathrm{~dB}$ \\
\hline & & $\begin{array}{l}\text { Amplificador } \\
\text { EDFA }\end{array}$ & \\
\hline & & $\begin{array}{l}\text { Ganancias }=10-15 \\
\mathrm{~dB}\end{array}$ & \\
\hline & & $\mathrm{NF}=4$ & \\
\hline
\end{tabular}

Fuente: elaboración propia

Tabla 2: Tramos de red.

\begin{tabular}{|r|l|l|l|}
\hline Tramo & Punto Inicial & Punto Final & Población \\
\hline 1 & Pto. Principal & Pto. de distribución 1 & P1 \\
\hline 2 & Pto. de distribución 1 & P2 & P2 \\
\hline 3 & Pto. de distribución 1 & Pto. de distribución 2 & P3 \\
\hline 4 & Pto. de distribución 2 & P4 & P4 \\
\hline
\end{tabular}

Fuente: elaboración propia

El plan de longitudes de onda expresado en frecuencias se muestra en la Tabla 3 .

El diseño de la red se inició con la realización de los presupuestos de potencia, de ancho de banda por tramo óptico y la elaboración de los puntos de distribución.
Tabla 3: Plan de frecuencias para el diseño.

\begin{tabular}{|l|l|}
\hline Frecuencia & Población \\
\hline 192.8 & P1 \\
\hline 192.9 & P2 \\
\hline 193 & P3 \\
\hline 193.1 & P4 \\
\hline 193.2 & Auxiliar1 \\
\hline 193.3 & Auxiliar2 \\
\hline
\end{tabular}

Fuente: elaboración propia

\subsection{Presupuesto de potencia y ancho de banda}

Teniendo en cuenta lo anterior, se realiza el cálculo del presupuesto de potencia para cada tramo en cada 
ruta $y$, en general, se busca que para cada población haya una potencia de recepción entre $-10 \mathrm{dBm}$ a -20 $\mathrm{dBm}$ con una potencia de trasmisión de $15 \mathrm{dBm}$ desde el punto de distribución principal.

La fórmula general para el cálculo del presupuesto de potencia es:

$P_{T x} d B m+G_{A o} d B-L_{T T} d B-M D d B-L_{i n} d B=S_{R x} d B m$

Donde:

$P_{T x}=$ potencia de transmisión en $\mathrm{dBm}$.

$G_{A o}=$ ganancia adicionada en el tramo.

$L_{T T}=$ perdidas totales en la trayectoria.

$M D=$ margen de diseño.

$L_{i n}=$ perdidas por inserción (dependen de cada tramo en cuestión).

$S_{R x}=$ sensibilidad del receptor.

Además, las pérdidas totales en la trayectoria se definen:

$$
L_{T T} d B=\alpha_{F_{o}} * l_{F_{o}}+\alpha_{c} * N C+\alpha_{E} * N E
$$

Donde:

$\alpha_{F_{o}}=$ atenuacion de la fibra óptica en $\mathrm{dB} / \mathrm{km}$

$l_{F o}=$ longitud de la fibra óptica en $\mathrm{km}$

$\alpha_{c}=$ atenuación de los conectores en $\mathrm{dB}$

$N C=$ número de conectores en el tramo.

$\alpha_{E}=$ atenuación de los empalmes en dB

$N E=$ numero de conectores.

El número de conectores se puede hallar:

$$
N E=\left(\frac{l_{f o}}{l_{c f o}}-1\right)
$$

Donde:

$l_{c F_{o}}=$ longitud de carrete de fibra óptica en $\mathrm{km}$.

Para el cálculo del presupuesto del ancho de banda se considera la modulación NRZ, por lo que se define:

$$
B_{\text {proy }} G H z=\frac{1}{2} * f_{b}
$$

Donde:

$f_{b}=$ tasa de transmisión.

Entonces para una tasa de transmisión de 10 Gbps contemplados para el diseño se tiene:

$$
B_{\text {proy }}=\frac{10}{2}=5 G H z \cdot d e
$$

Por lo tanto, al momento de calcular cualquier tramo de red este, por lo menos, debe tener un ancho de banda de $5 \mathrm{GHz}$ o superior.

Considerando el resultado anterior, se tiene que para un tramo de la red se define:

$$
T_{s}=T_{S F o}=\sqrt{T_{S M O D A L}^{2}+T_{S C R O M A T I C O}^{2}+T_{S P M D}^{2}}
$$

Donde:

$T_{S}=$ tiempo de subida

$T_{S F O}=$ tiempo de subida de la fibra óptica

Para el diseño contemplado y debido que la fibra es monomodo el $T_{S M O D A L}=0$ y dado que la tasa de transmisión diseñada no es superior a los 10 Gbps se considera que $T_{S P M D}=0$.

Por lo tanto, la ecuación (6) queda de la siguiente forma:

$$
T_{s}=T_{S F o}=\sqrt{T_{S C R O M A T I C O}^{2}}=T_{S C R O M A T I C O}
$$

y se define:

$$
B_{G H z}=\frac{0,35}{T_{S F o}} \geq B_{\text {proy }}
$$

Por lo que, de acuerdo con las ecuaciones anteriores, se tiene que en cuanto al presupuesto de ancho de banda los siguientes resultados (Tabla 4 ).

Tabla 4: Dispersión cromática para cada tramo de red.

\begin{tabular}{|c|l|l|c|l|l|}
\hline Tramo & Origen & Destino & $\begin{array}{l}\text { Distancia } \\
(\mathbf{k m})\end{array}$ & $\begin{array}{l}\text { Disper. } \\
(\mathbf{p s})\end{array}$ & $\begin{array}{l}\text { Perdi. } \\
(\mathbf{d B})\end{array}$ \\
\hline 1 & Pto. Principal & $\begin{array}{l}\text { Pto de distribución } \\
1\end{array}$ & 65 & 1105 & 4.47006 \\
\hline 2 & $\begin{array}{l}\text { Pto de } \\
\text { distribución 1 }\end{array}$ & P2 & 57 & 969 & 4.12553 \\
\hline 3 & $\begin{array}{l}\text { Pto de } \\
\text { distribución 1 }\end{array}$ & $\begin{array}{l}\text { Pto de distribución } \\
2\end{array}$ & 35 & 595 & 3.17809 \\
\hline 4 & $\begin{array}{l}\text { Pto de } \\
\text { distribución 2 }\end{array}$ & P4 & 15 & 255 & 2.31678 \\
\hline
\end{tabular}

Fuente: elaboración propia 
Las pérdidas provocadas por la adición de las fibras de compensación por tramo fueron calculadas linealizando los parámetros de atenuación cuando se compensa una fibra G.652.D, dichos parámetros se encuentran con el módulo de fibra compensadora Draka en [12].

Para garantizar un ancho de banda de 10 Gbps se debe tener una dispersión cromática máxima de:

$$
T_{s}=\frac{0,35}{5 G H z}=0,07 n s=70 p s d e
$$

Por esta razón se debe adicionar, en cada uno de los tramos, fibras compensadoras de dispersión. Teniendo en cuenta lo anterior, en el presupuesto de potencia se tiene lo evidenciado en la Tabla 5.

Tabla 5: Presupuesto de potencia de la red.

\begin{tabular}{|c|c|c|c|c|c|c|c|c|c|}
\hline $\begin{array}{c}\text { Tram } \\
\mathbf{0}\end{array}$ & $\begin{array}{c}\text { Empalme } \\
\mathbf{s}\end{array}$ & $\begin{array}{c}\text { Perdi } \\
(\mathbf{d B})\end{array}$ & $\begin{array}{c}\text { PRx } \\
(\mathbf{s i n} \\
\mathbf{a m p l i})\end{array}$ & $\begin{array}{c}\text { PRx } \\
(\mathbf{c o n} \\
\mathbf{c o m p})\end{array}$ & $\mathbf{A m p}$ & $\begin{array}{c}\text { PRx } \\
\text { (comp+am } \\
\mathbf{p})\end{array}$ & $\begin{array}{c}\text { PTx } \\
\text { dest }\end{array}$ & $\begin{array}{c}\text { PRx } \\
\text { pob. }\end{array}$ & $\begin{array}{c}\text { aten. } \\
\text { Adicio }\end{array}$ \\
\hline 1 & 12 & 4.47 & -6.40 & -11.07 & 10 & -1.27 & -9.27 & -13.27 & 1.8 \\
\hline 2 & 11 & 4.13 & -21.97 & -26.30 & 15 & -11.50 & & -15.50 & 1.7 \\
\hline 3 & 6 & 3.18 & -17.07 & -20.45 & 15 & -5.65 & -13.65 & -17.65 & 1.2 \\
\hline 4 & 2 & 2.32 & -17.05 & -19.56 & 10 & -9.76 & & -13.76 & 0.8 \\
\hline
\end{tabular}

Fuente: elaboración propia

Donde:

Perdi: corresponde a las pérdidas que introduce la adición de la fibra compensadora de dispersión y cuyos valores se obtuvieron de [12].

PTx dest: potencia de transmisión de un punto destino al siguiente.

PRx pob: potencia de recepción que recibe un usuario o elemento de distribución a hogares en una población.

Aten. Adicio: atenuación adicional que se agrega al momento de simular producida por el uso de conectores y empalmes en cada tramo óptico.

Además, se resalta que en cada uno de los tramos se tuvieron en cuenta el uso de seis conectores (dos por cada elemento) y no se utilizó margen de diseño, ya que este es usado en el momento de la implementación física, pero para efectos teóricos no tiene mucho sentido, aunque el software utilizado introduce un poco de ruido simulado.

\subsection{Diseño de puntos de distribución}

Como se mencionó anteriormente, en este diseño en específico se utilizaron switches ópticos y arreglos de guías de onda AWG, por lo que se usó un esquema de múltiples longitudes de onda. El diseño cuenta con tres puntos de distribución, el punto de distribución principal es básicamente donde se encuentra el transmisor para cada una de las poblaciones (Figura 6, Figura 7 y Figura $8)$.

Figura 6: Punto de distribución principal.

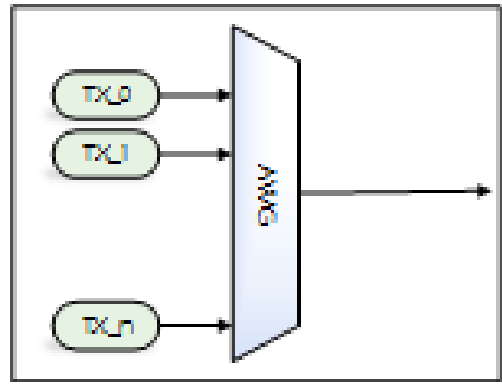

Fuente: elaboración propia.

Figura 7: Punto de distribución 1.

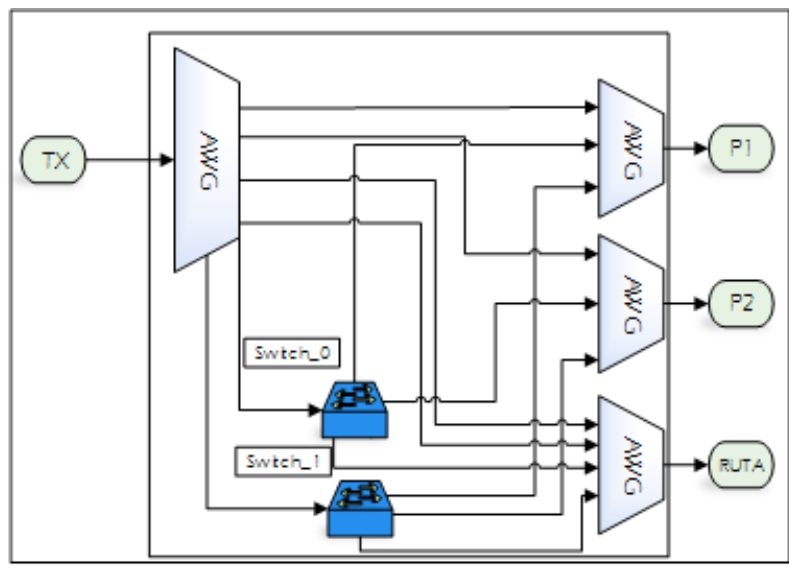

Fuente: elaboración propia.

Figura 8: Punto de distribución 2.

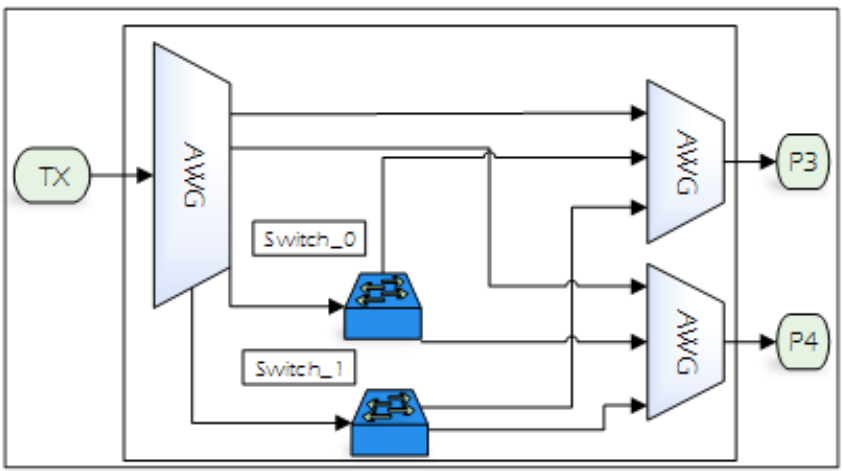

Fuente: elaboración propia. 
Los puntos de distribución secundarios están diseñados para redireccionar cualquiera de las dos longitudes de onda de respaldo hacia cualquier destino con la configuración correcta de los switches, para lo cual existen las siguientes tablas de enrutamiento de longitud de onda (Tabla 6 y Tabla 7).

Tabla 6: Enrutamiento punto de distribución 1.

\begin{tabular}{|c|c|c|}
\hline bit 1 & bit 0 & Destino \\
\hline $\mathrm{X}$ & 0 & $\mathrm{P} 1$ \\
\hline 1 & 0 & $\mathrm{P} 1$ \\
\hline 0 & 1 & $\mathrm{P} 2$ \\
\hline 1 & 1 & Tramo 3 \\
\hline
\end{tabular}

Fuente: elaboración propia

Tabla 7: Enrutamiento punto de distribución 2.

\begin{tabular}{|c|c|}
\hline bit 0 & Destino \\
\hline 0 & $\mathrm{P} 3$ \\
\hline 1 & $\mathrm{P} 4$ \\
\hline
\end{tabular}

Fuente: elaboración propia

Estas tablas aplican para todos los switches dentro de un punto de distribución (cada switch óptico maneja solo una longitud de onda); además, hay que tener en cuenta que existe una relación entre las tablas ya que, en este diseño, si una longitud de onda es usada en las poblaciones P1 o P2 - las cuales son controladas desde el primer punto de distribución-, no podrán ser usadas en las poblaciones P3 y P4, independientemente de los valores en sus tablas de enrutamiento. Por otro lado, si una longitud de onda es usada en una población no podrá emplearse en otro lugar al mismo tiempo.

\section{Simulación}

Para la simulación de la red propuesta se utilizó el software Optisystem de la empresa Optiwave Design Software, el cual permite simular el diseño de redes ópticas bajo ciertas características y está enfocado al diseño de redes ópticas de última generación.

Esta red fue simulada con los parámetros encontrados en la Tabla 1 y los puntos de distribución de acuerdo con la Figura 6, la Figura 7 y la Figura 8. Cabe destacar, como se nombró anteriormente, que los elementos atenuadores en cada tramo óptico representan a los conectores y empalmes. Finalmente, para poder observar los espectros de cada longitud de onda se utilizaron analizadores de espectro en cada receptor y para obtener el diagrama de ojo se utilizó un regenerador $3 \mathrm{R}$ con el fin de visualizarlo en un analizador de BER.

Figura 9: Simulación de la red diseñada.

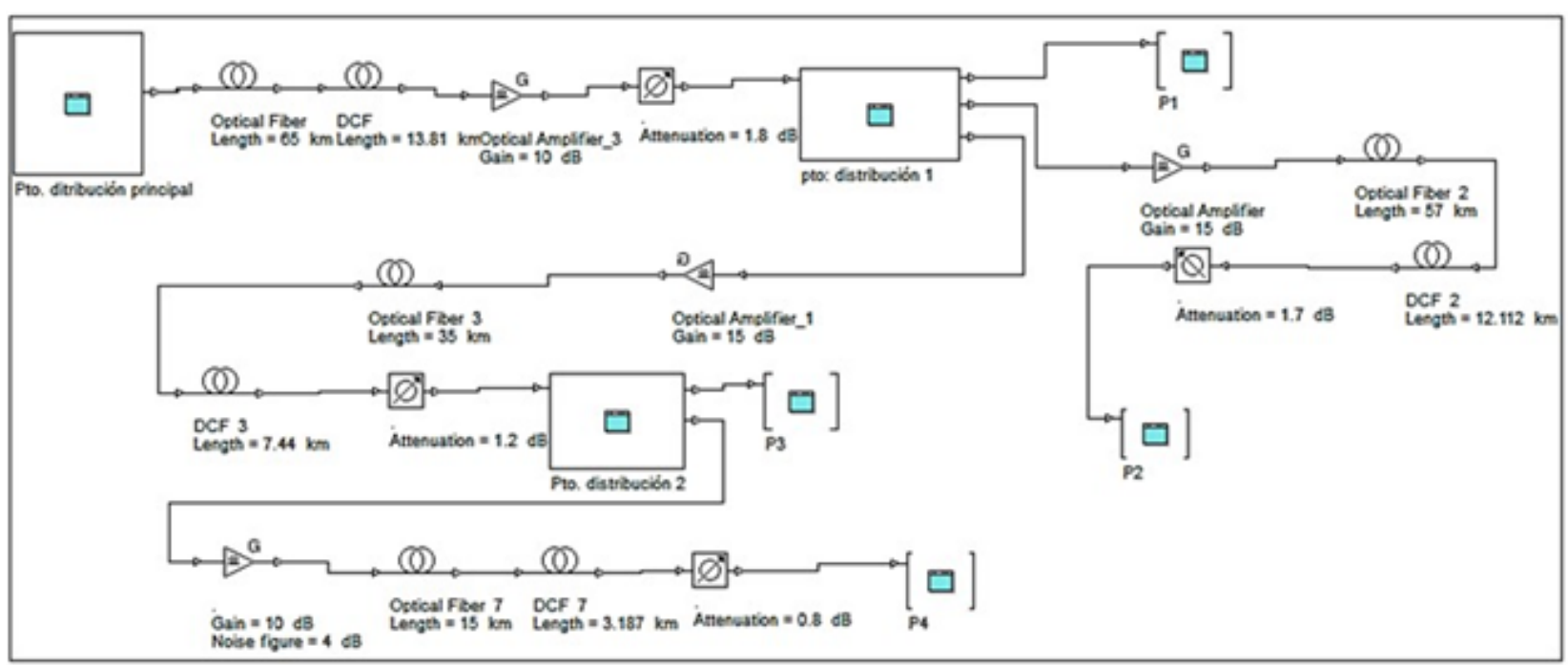

Fuente: elaboración propia.

La configuración de los puntos de distribución quedó de tal forma que la longitud de onda auxiliar 1 llegará a la población P1 y la longitud de onda auxiliar 2 a la población P3 (Figura 9).

\section{Resultados}

En cuanto a potencia de las señales recibidas se tiene lo expuesto en la Tabla 8 y la Figura 10. 
Tabla 8: Parámetros de las señales recibidas en cada una de las poblaciones.

\begin{tabular}{|c|c|c|c|c|c|}
\hline $\begin{array}{c}\text { Frecuencia } \\
(\mathbf{T H z})\end{array}$ & $\begin{array}{c}\text { Long. de onda } \\
(\mathbf{n m})\end{array}$ & $\begin{array}{c}\text { Potencia } \\
(\mathbf{d B m})\end{array}$ & $\begin{array}{c}\text { Potencia } \\
(\mathbf{W})\end{array}$ & $\begin{array}{c}\text { Potencia de ruido } \\
(\mathbf{d B m})\end{array}$ & $\begin{array}{c}\text { OSNR } \\
(\mathbf{d B})\end{array}$ \\
\hline 192.8 & 1554.9401 & -13.6792 & $4.29 \mathrm{E}-05$ & -59.049889 & 45.3707 \\
\hline 192.9 & 1554.134 & -15.851779 & $2.60 \mathrm{E}-05$ & -57.637667 & 41.7859 \\
\hline 193 & 1553.3288 & -18.090049 & $1.55 \mathrm{E}-05$ & -59.871295 & 41.7812 \\
\hline 193.1 & 1552.5244 & -14.21086 & $3.79 \mathrm{E}-05$ & -51.82133 & 37.6105 \\
\hline 193.2 & 1551.7208 & -14.397725 & $3.63 \mathrm{E}-05$ & -58.805573 & 44.4078 \\
\hline 193.3 & 1550.918 & -19.605465 & $1.10 \mathrm{E}-05$ & -60.997713 & 41.3922 \\
\hline
\end{tabular}

Fuente: elaboración propia
Como se puede analizar respecto a las gráficas anteriores, las señales llegan con una potencia adecuada a cada uno de sus destinos, poseen bajo ruido menor a $-50 \mathrm{dBm}$ y una relación señal a ruido alta mayor a $37 \mathrm{~dB}$, lo cual es lo que se quiere obtener; sin embargo, estos valores aún no son concluyentes, ya que, independiente de sí la potencia de la señal recibida sea la adecuada o no, si no hubo un control de la dispersión no se podrían obtener los datos (Tabla 9).

Figura 10: Espectros ópticos de las longitudes de onda recibidas.

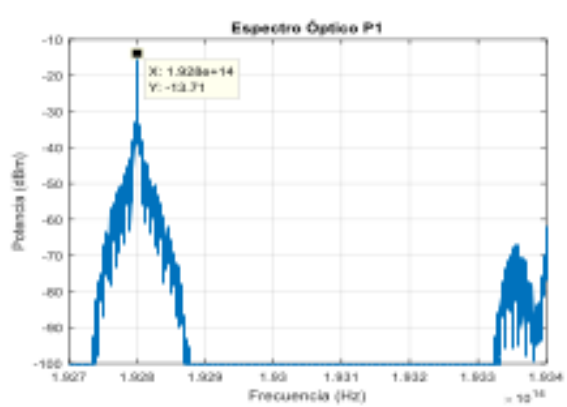

a) Espectro óptico en P1 192.8 (THz)

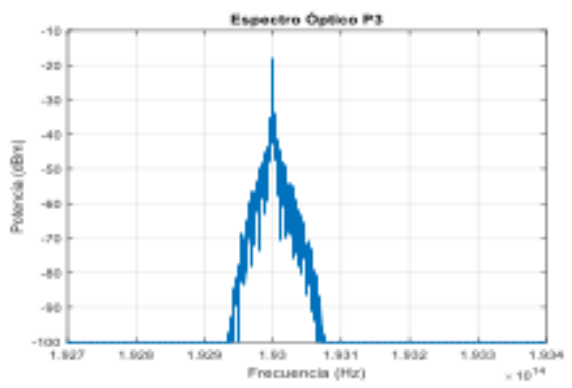

c) Espectro óptico en P3 193 (THz)

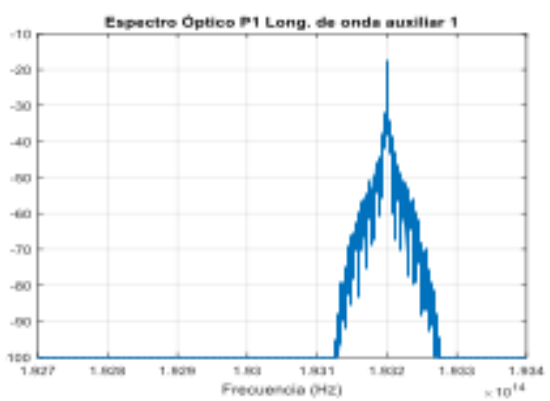

:) Espectro óptico en P1 longitud de onda auxiliar 193.2 (THz)

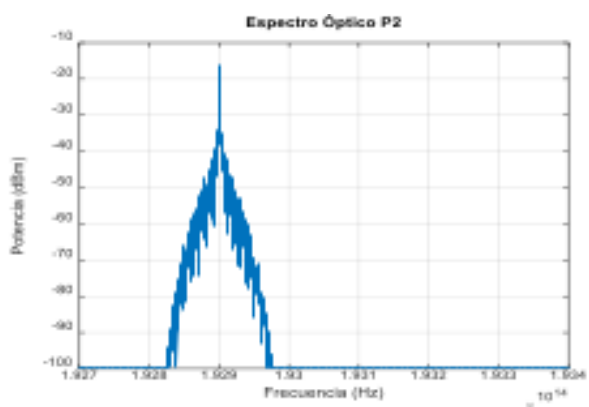

b) Espectro óptico en P2 192.9 (THz)

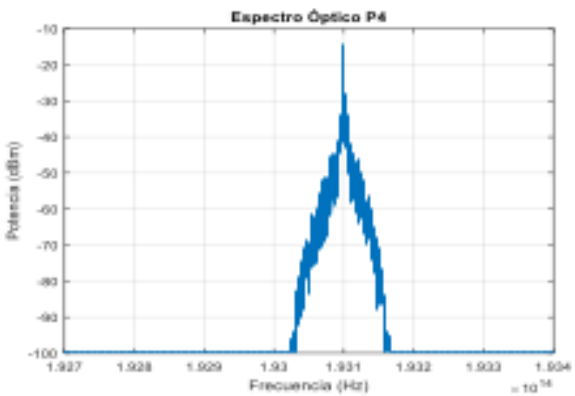

d) Espectro óptico en P4 193.1 (THz)

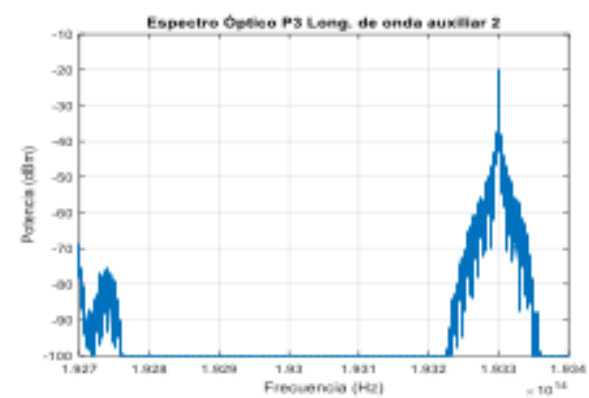

f) Espectro óptico en P3 longitud de onda auxiliar $193.3(\mathrm{THz})$

Fuente: elaboración propia. 
Tabla 9: Valores de analizador de espectro por longitud de onda

\begin{tabular}{|l|c|r|r|r|r|r|}
\hline Población & $\begin{array}{l}\text { Frecuencia } \\
\text { (THz) }\end{array}$ & Factor Q & Min. BER & Eye Height & Threshold & Decisión Inst. \\
\hline P1 & 192.8 & 30.2439 & $2.39 \mathrm{E}-201$ & 0.0002453 & $4.41 \mathrm{E}-05$ & 0.621094 \\
\hline P2 & 192.9 & 17.9858 & $9.87 \mathrm{E}-73$ & 0.0001331 & $2.72 \mathrm{E}-05$ & 0.628906 \\
\hline P3 & 193 & 15.7505 & $2.86 \mathrm{E}-56$ & $7.90 \mathrm{E}-05$ & $2.33 \mathrm{E}-05$ & 0.636719 \\
\hline P4 & 193.1 & 15.7505 & $2.86 \mathrm{E}-56$ & $7.90 \mathrm{E}-05$ & $2.33 \mathrm{E}-05$ & 0.636719 \\
\hline P1 & 193.2 & 25.8797 & $4.46 \mathrm{E}-148$ & 0.0002005 & $4.06 \mathrm{E}-05$ & 0.625 \\
\hline P3 & 193.3 & 14.6505 & $6.02 \mathrm{E}-49$ & $5.35 \mathrm{E}-05$ & $1.93 \mathrm{E}-05$ & 0.480469 \\
\hline
\end{tabular}

Fuente: elaboración propia

A partir de la Figura 11 se puede visualizar que de todas las longitudes de ondas que llegan a las poblaciones, el diagrama de ojo en P1 es más definido, mientras que para los demás poblaciones el diagrama de ojo tiene cierta distorsión y esto se debe a que a mayor distancia recorrida la señal se ve más expuesta a efectos no lineales que introduce la fibra; sin embargo, en los resultados obtenidos el factor de calidad es superior a 14.65 y el BER (Bit Error Rate) es bajo, ya que es menor a $1 \times 10^{-12}$ en todas las poblaciones, lo cual es una sugerencia encontrada en la recomendación b-ITU-T G-Sup.39 [13]. Para recuperar las señales en cada uno de los receptores de manera satisfactoria, además, se puede comparar que las longitudes de onda auxiliares no difieren significativamente en su diagrama de ojo a las fijas en una misma población.

Figura 11: Diagramas de ojo en cada una de las poblaciones.

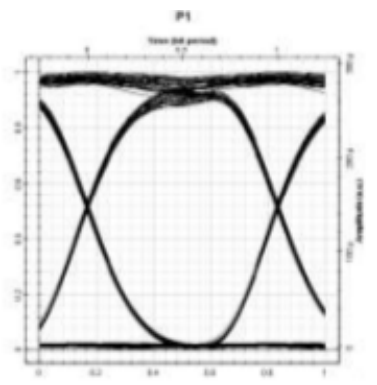

a)

Diagrama de ojo P1 192.8 (THz)

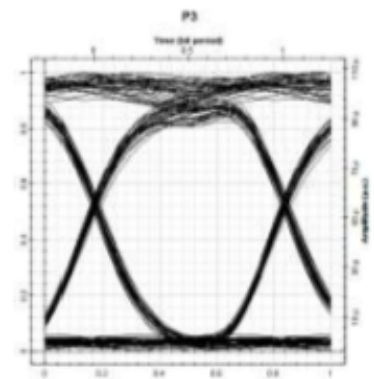

c)

Diagrama de ojo P3 193 (THz)

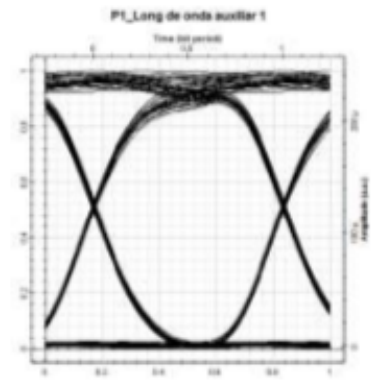

)

Diagrama de ojo P1 longitud de onda auxiliar 1193.2 (THz)

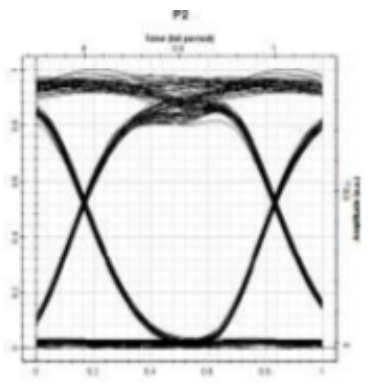

b)

Diagrama de ojo P2 192.9 (THz)

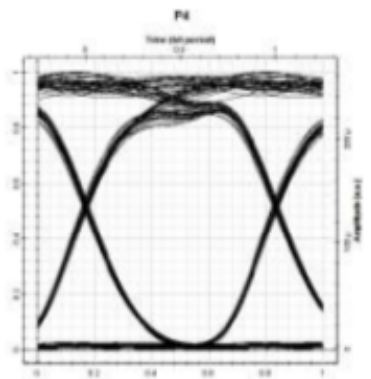

d)

Diagrama de ojo P4 193.1 (THz)

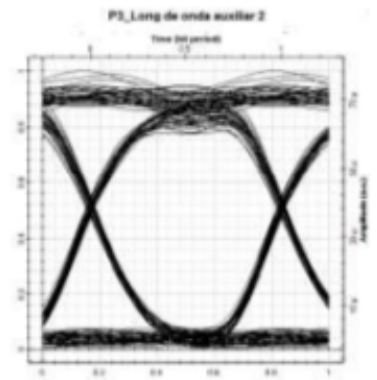

Diagrama de ojo P3 longitud de onda auxiliar $2193.3(\mathrm{THz})$

Fuente: elaboración propia. 


\section{Conlusiones}

Existen desafíos en este tipo de implementaciones, ya que, se debe definir cómo funcionará el receptor, porque si bien es cierto que en el diseño propuesto en cada población se pueden usar hasta tres longitudes de onda, en el punto final deben existir al menos tres receptores cada uno sintonizado con la longitud de onda correspondiente o, en su defecto, debe haber un receptor con la capacidad de sintonizarse a cualquier longitud de onda, los cuales son denominados "Colorless" y aún se encuentran en investigación.

Como se logra observar, el sistema propuesto es fácilmente escalable a una mayor cantidad de tráfico, ya que solo es necesario cambiar el tamaño de los AWG para manipular una mayor cantidad de longitudes de onda y, por ende, un mayor ancho de banda en la red.

Un aumento en la cantidad de longitudes de onda no afecta de gran manera el presupuesto de potencia y de ancho de banda inicialmente calculado, aunque es cierto que diferentes longitudes de onda en un mismo sitio tendrán variaciones, se espera que estas no sean de más de $2 \mathrm{~dB}$ respecto a las otras, lo cual, es una de las ventajas principales del AWG y que permite un grado de escalabilidad mayor aprovechando el mismo tendido de fibra óptica.

Los valores de tasa de error de bit (BER) obtenidos son más que suficientes para garantizar la tasa de transmisión de 10 Gbps propuesta inicialmente en el diseño, sin embargo, si se quiere escalar a una tasa de transmisión mayor, por ejemplo, 40 Gbps o más, se deben considerar efectos no lineales adicionales aparte de la dispersión cromática e incluso manejar diversos tipos de modulación diferentes a NRZ.

Se deben experimentar con diversas configuraciones de amplificadores ópticos y esquemas de compensación, ya que, si bien los cálculos realizados no varían, estos cambios pueden modificar significativamente la forma del diagrama de ojo y, por ende, la tasa de error de bit, ya sea para mejorar o para empeorar.

\section{Referencias}

[1] Internet World Stats, "Internet Usage and World Population Statistics, Miniwatts Marketing Group". [En línea]. Disponible en: http://www. internetworldstats.com/stats.htm\#links.

[2] Cisco System Inc., "The Zettabyte Era: Trends and Analysis". [En línea]. Disponible en: http://www.cisco.com/c/en/us/ solutions/collateral/service-provider/

visual-networking-index-vni/VNI_

Hyperconnectivity_WP.html.

[3] H. Song, B. Kim y B. Mukherjee, "Long-reach optical access networks: A survey of research challenges, demonstrations, and bandwidth assignment mechanisms", IEEE Communications Surveys and Tutorials, vol. 12, $\mathrm{n}^{\circ}$. 1, pp. 112-123, 2010. https: //doi.org/10.1109/SURV.2010.020110.00040

[4] D. Shea y J. E. Mitchell, "Architecture to integrate multiple PONs with long reach DWDM backhaul", IEEE Journal on Selected Areas in Communications, vol. 27, n. 2, p. 126-133, 2009. https://doi.org/ 10.1109/JSAC. 2009.090204

[5] S. F. Sha, M. Khan y M. K. Shahid, "Reach extendibility of passive optical network technologies", Optical Switching and Networking, vol. 18, $\mathrm{n}^{\circ} .3$, pp. 211-221, 2015. https://doi.org/10.1016/j.osn. 2015.07 .004

[6] A. M. Ragheb y H. Fathallah, "Performance analysis of next generation-PON architectures", 8th International Conference on High-Capacity Optical Networks and Emerging Technologies, pp. 339-345, 2011.

[7] T. C. Tavares, "Análise do Desempenho de Amplificadores Ópticos Semiconductores Reflectivos em Redes Ópticas de Acesso da Próxima Geração", Tesis de maestría, Universidade de Coimbra, Portugal, 2014.

[8] I. V. De Voorde, C. M. Martin, I. Vandewege y X. Z. Oiu, "The SuperPON Demonstrator: An Exploration of Possible Evolution Paths for Optical Access Networks", IEEE Communications Magazine, vol. $38, \mathrm{n}^{\circ} .2$, pp. 74-82, 2000. https://doi .org/10. $1109 / 35.819899$

[9] G. Talli y P. D. Townsend, "Hybrid DWDM-TDM Long-Reach PON for Next- Generation Optical", Lightwave Technology, Journal, vol. 24, $\mathrm{n}^{\circ}$. 7, pp. 2827-2834, 2006. https://doi.org/10.1109/JLT. 2006.875952

[10] J. A. Lazaro, J. Prat, P. Chanclou, G. M. Tosi, A. Teixeira,I. Tomkos, R. Soila y V. Koratzinos, "Scalable extended reach PON", Conference on Optical Fiber Communication, pp. 1-3, 2008. https: //doi.org/10.1109/OFC. 2008.4528488

[11] R. Ramaswani, S. Kumaer y S. Galen, "Optical Network a Practical Perspetive", Estados Unidos: Elseiver, 2010. 
[12] Draka, "Prysmian Group". [En línea]. DrakaElite---DCM-Family_0.pdf

Disponible en: https://www.prysmiangroup. $\mathrm{com} /$ sites/default/files/business_ markets/markets/downloads/datasheets/

[13] International Telecommunication Union, "Optical system design and engineering considerations", Suiza: ITU-T 2016. 